\title{
Tipos de estructuras sociales agrarias en la formación social argentina. Un análisis a nivel departamental: 2001-2002
}

\author{
Types of agrarian social structures in Argentinian social formation. A county level analysis: 2001-2002
}

\author{
Germán Rosati \\ Instituto de Altos Estudios Sociales - Universidad Nacional de San Martin. CONICET. \\ Programa de Investigaciones sobre el Movimiento de la Sociedad Argentina (PIMSA), \\ Argentina \\ german.rosati@gmail.com
}

\author{
Adriana Chazarreta \\ Instituto de Altos Estudios Sociales - Universidad Nacional de San Martín. CONICET, \\ Argentina \\ adchazarreta@gmail.com
}

\begin{abstract}
Resumen:
El presente trabajo busca identificar las diferentes estructuras sociales agrarias que componen la formación social argentina. Se utilizan fuentes censales (agropecuarias y de población) a nivel departamental y se realiza un análisis de clustering. Como resultado, se identifican cuatro estructuras agrarias: 1) alto grado de desarrollo de relaciones salariales; 2) alto grado de desarrollo de relaciones salariales y existencia de superpoblación latente; 3) alto desarrollo de relaciones salariales articulada con fracciones de pequeños propietarios simples y acomodados, y 4) pequeña producción mercantil simple con elementos de descomposición. Cada una se caracteriza por diferentes proporciones de tipos de explotaciones y grupos sociales.
\end{abstract}

Palabras Clave: Estructura agraria, Clustering, Desarrollo capitalista.

\section{Abstract:}

This paper tries to identify the different agrarian social structures in Argentina's social formation. Census sources (agricultural and population) are used at the departmental level and a clustering analysis is performed. As a result, four agrarian structures are identified: 1) high development degree of wage relationships, 2) high development degree of wage relationships and existence of latent surplus population, 3) high development degree of wage relationships with fractions of small simple and 4) small mercantile production and small mercantile production with decomposition elements. Each is characterized by different proportions of types of farms and social groups.

KEYwORDS: Agrarian structure, Clustering, Capitalist development.

\section{INTRODUCCIÓN ${ }^{1}$}

Desde hace aproximadamente tres décadas, se profundiza el desarrollo capitalista en el campo argentino. Dicha profundización se enmarca, al igual que otras actividades económicas, en transformaciones vinculadas con la desregulación económica (con el retraimiento del Estado en sus funciones reguladoras), la apertura económica (transnacionalización del mercado de insumos e importante presencia del capital financiero) y la innovación tecnológica. En ese contexto se produjeron dos grandes procesos: la agriculturización y la sojización de la producción agropecuaria.

El primer proceso comenzó hacia la década de los 60 e implicó el predominio del reemplazo de las actividades ganaderas por las actividades vinculadas a la agricultura, con lo que se produjo un enorme crecimiento del volumen físico de la producción agrícola. El segundo proceso se refiere a la sustitución de 
otros cultivos y de la actividad pecuaria por la soja (Rosati, 2013). Paralelamente, se produjo un gran aumento de la productividad agraria, asociada principalmente a la introducción en el país de semillas mejoradas (por ejemplo, de trigo, soja, maíz y girasol). Esto se afianzó en 1996 al liberalizarse el mercado de semillas transgénicas, y el país pasó a ocupar el segundo puesto en 2001 como exportador mundial de cultivos transgénicos. Por su parte, la soja (aunque este modelo se replica total o parcialmente en otros cultivos) se basa en la aplicación de un paquete tecnológico que consta de insumos variados como las semillas (genéticamente modificadas), agroquímicos y maquinaria especializada para la siembra directa (que no necesita laboreo de campo), y requiere para su implementación de superficies de grandes escalas. Asimismo, los cambios también se verifican a nivel de la gestión, pues esta se redefinió a partir de la incorporación de nuevas tecnologías de comunicación e información, en las que prevalecen la innovación empresarial, la visión global y la flexibilidad de procesos. Además, el complejo sojero destina masivamente su producción a la exportación, y ocupa un lugar importante en el total de exportaciones y en el aporte de divisas al país.

Si bien estos procesos tienen su epicentro en la región pampeana, no son exclusivos de ella; por el contrario, se extienden a diversas regiones del país. Una de las formas (no la única) de difusión en que dichos cambios se producen es a través de la expansión de los cultivos pampeanos (como soja, maíz y trigo) a otras zonas. Esta expansión puede tomar varias modalidades; por ejemplo, la incorporación de áreas nuevas a la producción o a través de la sustitución de cultivos existentes (Rosati, 2013). Además, existen otros procesos de cambio en la estructura agraria argentina que corrieron paralelamente a los señalados. En efecto, ciertas economías agrarias provinciales no desaparecieron ni fueron reemplazadas sino que sufrieron movimientos específicos de reconversión y modernización (como la vitivinicultura en Mendoza y San Juan), y otras han sido desplazadas por cultivos diferentes de los cultivos pampeanos (como es el caso de Misiones, donde se produce el desplazamiento de la actividad tradicional -la yerba mate- por la forestal) (Chazarreta, Poth y Ramírez, 2015).

Este trabajo se enmarca en un proyecto más general que busca identificar la forma en que dichos procesos de profundización de las relaciones capitalistas en el campo se vinculan con los diferentes tipos de estructuras sociales agrarias que existen al interior de la formación social argentina. Constituye, en ese sentido, una primera aproximación de carácter exploratorio a la identificación de las diferentes estructuras que conforman el campo argentino. A su vez, dado que se trata de una aplicación sobre los últimos datos disponibles, confiables y comparables (Censo Nacional de Población, Hogares y Vivienda -CNPyV- 2001 y Censo Nacional Agropecuario -CNA- 2002), el interés de este trabajo radica, también, en el diseño de una metodología de análisis para aplicar a censos más recientes. 2

Para Margiotta y Benencia (1988) tres son los componentes básicos de la estructura agraria: estructura de la tenencia de la tierra (cómo se distribuye, quiénes son sus propietarios y cómo se controla su producción), estructura económico-productiva (qué, cómo y para qué se produce, lo cual será central para establecer las diferentes formas productivas y las formas de acumulación) y la estructura social (los colectivos que participan de la estructura agraria y el entramado de relaciones sociales que conforman la dinámica social).

Este trabajo pretende enfocarse específicamente en el último componente (la estructura social), pero llegando a un nivel de desagregación departamental (el máximo permitido por la información disponible). Esta desagregación permitirá a su vez proponer una nueva agrupación de áreas, basada en un nuevo criterio no asociado necesariamente a características geográficas como distancia o vecindad. No obstante, queda pendiente el análisis de otras determinaciones, como la forma de tenencia de la tierra, el uso del suelo y su composición.

La identificación de las diferentes estructuras agrarias en la Argentina se vincula de forma estrecha con el problema de las llamadas "vías del desarrollo del capitalismo agrario". La literatura clásica ha descrito y analizado tres grandes formas en que se produce dicho desarrollo. En primer lugar, la descripta por Marx (2005), cuyo referente empírico fundamental es la expansión de las relaciones salariales en el campo británico 
y que identifica tres grandes figuras que componen la estructura agraria: los terratenientes (propietarios de la tierra y perceptores de renta), los arrendatarios (dueños del capital) y los trabajadores asalariados.

La investigación empírica posterior mostró la fortaleza de sus categorías teóricas, en tanto permitía identificar formas "híbridas" de desarrollo, es decir, formadas por diferentes combinaciones de las figuras analizadas por Marx. Así, la segunda forma de desarrollo del capitalismo en el campo es la "vía junker", estudiada por Lenin $(1983,1975)$. Esta provee un ejemplo de otro tipo de estructura agraria (y otro proceso de desarrollo histórico), en el cual fueron los grandes terratenientes los que dinamizaron la expansión del capitalismo agrario y reunieron las figuras del terrateniente y el arrendatario capitalista. De forma similar, la tercera y última forma, denominada "vía farmer" (Lenin, 1985), tuvo como rasgo central una estructura social caracterizada por productores de escala pequeña que reunían a las tres figuras del modelo inglés: la tierra y el capital en propiedad y el uso de trabajo familiar (y ocasionalmente asalariado). Al mismo tiempo, el análisis del caso ruso (Lenin, 1975) permitió identificar formas específicas de expansión de las relaciones salariales en el campo en contextos de relaciones serviles y los procesos de diferenciación social que ocurren como consecuencia. En todos estos ejemplos, se observa la conformación de diferentes estructuras sociales vinculadas a procesos divergentes de desarrollo del capitalismo en el campo.

La investigación sobre las modalidades de desarrollo capitalista en Argentina y la conformación de sus respectivas estructuras agrarias parece haber quedado restringida a una serie muy detallada de estudios que se focalizan en diferentes regiones del país. No obstante, la región pampeana suele aparecer sobrerrepresentada en estos análisis, muy particularmente en los debates en torno a la existencia o no de procesos de centralización de la propiedad agropecuaria (Pucciarelli, 1991; Barsky y Pucciarelli, 1997; Lodolla y Fossatti, 2004; Basualdo y Khavisse, 1993, Gras y Cáceres, 2017). Asimismo, estudios en muchas regiones del país se han concentrado en el análisis y descripción de la presencia y persistencia de estructuras sociales agrarias con alta prevalencia de pequeños productores mercantiles en sus diversas modalidades (Archetti y Stölen, 1975; Balsa, 2006; Bartolomé, 1975; Iñigo Carrera, 2011; Schiavoni, 1998). En ese sentido, el horizonte comparativo (implícito o explícito) parecen ser las similitudes y diferencias con la "via farmer". ${ }^{3}$ No obstante, al analizar la formación agraria argentina en su totalidad es necesario remarcar que en ella (al igual que en toda formación social agraria concreta) coexisten diferentes estructuras sociales con elementos diferentes. De esta forma, será necesario evaluar qué elementos de las diferentes estructuras agrarias típicas coexisten y se combinan en las diferentes estructuras concretas de la Argentina. ${ }^{4}$

Ahora bien, existe una diversidad de antecedentes en los cuales las regionalizaciones rurales o agrarias se construyen a partir de criterios vinculados a dimensiones estrictamente productivas. Para ello se utilizan variables como superficie dedicada al cultivo y/o a la ganadería (Taylor, 1948; Slutzky, 1968; Barsky, Pucciarelli y Barsky 1997), valor de la producción (Barsky, S/F; Bertamini, Bervejillo, Silva y Tommasino 2011) o aspectos asociados a la agroecología o a la aptitud de los suelos (Cirio, 1984; Gómez, Peretti, Pizarro y Cascardo, 1991). También en algunos se combinan varias dimensiones, como productos y sistemas de producción predominantes (CONADE-CFI, 1964), uso del suelo y valor de la producción (Secretaría de Agricultura, Ganadería y Pesca-PNUD, 1987) o superficie implantada con cultivos y forrajeras, existencias ganaderas, números de explotaciones con distintas orientaciones ganaderas y valor de la producción (Barsky, 1997). Otros tienen un componente, además, demográfico, pues incorporan variables como empleo y densidad de la población rural (Forni y Benencia, 1993), ${ }^{5}$ densidad de mano de obra permanente y absorción de mano de obra asalariada permanente (Bisio y Forni, 1980) o principal actividad económica de la población (Vicien y Dejean del Castillo, 1948).

Si bien no todos los estudios anteriores cubren el total del país, ya que algunos se refieren a la zona de la pampa húmeda, se los señala en la medida que proponen algún criterio o metodología de regionalización o zonificación departamental. La mayor parte de estas investigaciones trabaja con datos de los censos nacionales agropecuarios y estadísticas de los productos y abarcan diferentes períodos, que van desde 1937 a 1988. 
En ese sentido, no parecen ser muy frecuentes los análisis que tengan como dimensión central la estructura agraria para la construcción de regiones o zonas de los departamentos de la República Argentina.

\section{DEFiniciones CONCEPTUALES Y FUENTES DE DATOS}

Para la identificación de las estructuras agrarias se utilizó información proveniente de dos fuentes secundarias de datos: el CNPyV del año 2001 y el CNA de 2002. El análisis se realizó a nivel de departamento. Alcanza la totalidad de las provincias de la Argentina donde se localice alguna actividad agropecuaria. En ambos casos, se trabajó con re-procesamientos de la información censal.

Con el CNPyV 2001 se construyeron distribuciones a nivel departamental de los grupos sociales fundamentales en la rama 1 (Agricultura, ganadería, silvicultura y pesca). Los grupos sociales refieren a conjuntos de seres humanos que se encuentran en una misma situación en la estructura económica respecto del conjunto de las relaciones de producción, cuya expresión jurídica son las relaciones de propiedad. Se consideran como dimensiones principales: a) la posición respecto de la propiedad o no propiedad de las condiciones y medios de vida y trabajo, y b) la función que representan en la producción y reproducción social, distinguiendo entre quienes cumplen o no funciones intelectuales de mando o auxiliares del grupo social dominante. Los criterios fundamentales para construir las distribuciones de los grupos presentadas se basan en los trabajos de Iñigo Carrera y Podestá (1985) y Donaire y Rosati (2009).

No obstante, debe tenerse en cuenta que estos grandes grupos constituyen categorías de carácter conceptual. Para llegar a la distribución presentada es necesario tomar una serie de decisiones operacionales para armonizar la información censal (en el caso de los grupos sociales fundamentales, censos de población y vivienda) con los conceptos más generales.

Para la construcción de las diferentes categorías de grupos sociales se utilizaron las variables censales de categoría ocupacional, carácter ocupacional y calificación de la ocupación. ${ }^{6}$

Así, es posible distinguir los siguientes grandes grupos fundamentales:

1. La gran burguesía y altos funcionarios constituyen la cúpula del capital; los terratenientes, los rentistas, etc., es decir, la personificación del gran capital, ya sea porque por su posición son sus propietarios o porque por su función constituyen el estado mayor que representa dichos intereses. Una aproximación operativa a este grupo se alcanza mediante las siguientes categorías censales: directivos y gerentes de categoría ocupacional "patrón” y "asalariado" ocupados en grandes empresas (es decir, empresas de más de 40 ocupados).

Los censos de población presentan problemas para captar a los rentistas en tanto constituyen (al menos en su forma pura) personas que solamente viven del cobro del alquiler de tierra bajo su propiedad. Este tipo de situaciones son clasificadas por la estadística oficial como población inactiva, de la cual no se releva mayor información en los censos de población.

2. La pequeña burguesía se encuentra constituida por propietarios de sus condiciones y medios de vida que no venden su fuerza de trabajo. Dentro de este grupo pueden diferenciarse dos subgrupos:

2. 1. La pequeña burguesía acomodada constituida por quienes en el sector agropecuario, además de ser propietarios de sus propias condiciones y medios de vida, son a su vez propietarios de los medios de vida y trabajo de otros. ${ }^{7}$ También forman parte quienes cumplen funciones de mando propias del capital aún bajo la forma del salario. Un acercamiento operativo al primer grupo de pequeños y medianos patrones puede realizarse a partir de la categoría censal de patrones, particularmente aquellos que dirigen establecimientos de hasta 40 personas. 
El segundo subgrupo, a su vez, puede ser cuantificado mediante la información censal de forma aproximada a partir de las categorías de directivos y gerentes asalariados de pequeñas y medianas empresas (es decir, de hasta 40 empleados). Además, se incluyen profesionales independientes y asalariados, y por extensión, quienes desempeñan funciones de asistencia técnico-profesional y quienes ejercen funciones superiores de apoyo administrativo, contable, jurídico y financiero.

2. 2. La pequeña burguesía pobre se encuentra constituida por quienes son sólo propietarios de sus propios medios de vida y trabajo. La operacionalización de este grupo se realiza mediante las categorías de pequeños propietarios del sector (generalmente, cuentapropistas), trabajadores independientes y similares, etc.

3. El proletariado y semiproletariado está constituido por quienes se encuentran expropiados total o parcialmente de sus condiciones de vida y de trabajo, y que, en consecuencia, se ven obligados a vivir principal o parcialmente de la venta de su fuerza de trabajo, ya sea que logren venderla o no. Es posible aproximarse a este grupo social mediante las categorías censales de asalariados (generalmente de calificación operativa y no calificada) y ciertas ocupaciones de los trabajadores por cuenta propia y familiares sin remuneración clasificadas como de calificación operativa y no calificada. Es decir, no se trata de un concepto directamente asimilable a la totalidad de la categoría censal de asalariados.

A su vez, para complementar la información proveniente del CNPyV, cuya unidad de análisis es la población, se ha incorporado información proveniente del Censo Nacional Agropecuario 2002. Particularmente, se utilizó la tipología de pequeños productores realizada en el marco del Instituto Interamericano de Cooperación para la Agricultura -IICA- (Obschatko, Foti y Román, 2007). ${ }^{8}$ Dicha tipología recurre a criterios como tamaño de la explotación, posesión de tractor, superficie irrigada y otros para construir tres tipos de productores hipotéticos:

(T1) un estrato superior de pequeño productor familiar capitalizado que (...) puede evolucionar (realizar una reproducción ampliada de su sistema de producción). No presenta en general rasgos de pobreza y sus principales carencias se refieren a servicios de apoyo a la producción (...);

(T2) un estrato intermedio de pequeño productor familiar (...) que posee una escasez de recursos (tierra, capital, etc.) tal que no le permite la reproducción ampliada o la evolución de su explotación, sino solamente la reproducción simple (es decir, mantenerse en la actividad), y presenta algunos rasgos de pobreza por falta de acceso a servicios sociales básicos; y

(T3) un estrato inferior de pequeño productor familiar, cuya dotación de recursos no le permite vivir exclusivamente de su explotación y mantenerse en la actividad (es 'inviable' en las condiciones actuales trabajando sólo como productor agropecuario), por lo que debe recurrir a otras estrategias de supervivencia (trabajo fuera de la explotación, generalmente como asalariado transitorio en changas y otros trabajos de baja calificación), posee acentuadas condiciones de pobreza, y su mantenimiento en el campo se explica, en una gran mayoría de casos, por el aporte que recibe de programas públicos de asistencia social y por otros ingresos eventuales.(Obschatko, Foti y Román, 2007, p. 36).

A su vez, todo el resto de las explotaciones queda clasificada como de "No pequeños productores". Estas explotaciones incluyen un abanico amplio de situaciones: desde pequeños productores que sí tienen uno o dos trabajadores no familiares remunerados permanentes -y por ello fueron excluidos de la definición de pequeño productor utilizada- hasta explotaciones empresariales -identificadas fundamentalmente por no ser familiares y poseer regímenes de tenencia de la tierra o formas jurídicas propias de una empresa-. En ese sentido, hay dos investigaciones (Obschatko, 2009 y Obschatko, Soverna y Tsakoumagkos, 2016) complementarias al estudio de pequeños productores, que abordaron parte de ese universo de explotaciones de "no pequeños 
productores" también a partir de datos del CNA 2002. Sin embargo, dado que en ninguno de los dos estudios se publicaron datos a nivel departamental, no fue posible incluirlos en el presente trabajo.

Tanto en el CNPyV como en el CNA se agregó para cada departamento la cantidad y la proporción de personas pertenecientes a la PEA, clasificadas en los diferentes grupos sociales fundamentales y la de explotaciones de cada tipo. Dada la baja cantidad de casos, se colapsó en una sola categoría a todos los patrones (los grupos "gran burguesía" y "pequeña burguesía acomodada" y "pequeños patrones").

Sobre la base de estos indicadores, se construyó una tipología de departamentos que logra una aproximación a las diferentes estructuras agrarias que coexisten en la formación social argentina. Se utilizaron dos técnicas para el análisis de datos: análisis de componentes principales (PCA, por sus siglas en inglés) y análisis de cluster.

\section{LA EVOLUCión DE LA ESTRUCTURA AGRARIA ARGENTINA A NIVEL AGREgADo}

En un trabajo reciente (Chazarreta y Rosati, 2016) se analizaron, a nivel agregado regional, las relaciones predominantes de producción y los grupos sociales en el agro argentino a partir de los datos provenientes de los censos de población (1991 y 2001).

Entre 1991 y 2001 descendió, entre la población ocupada en el agro, la posición de proletariado y semiproletariado, del $61 \%$ al $55 \%$. Este descenso se explica tanto por el crecimiento de la pequeña burguesía pobre (que pasa del $28 \%$ al $35 \%$ ) como por la importante expulsión de ocupados en el sector (entre esos años, la población total ocupada en el sector agropecuario descendió un $33 \%$ ). En cuanto a los sectores propietarios de sus condiciones de existencia y que contratan o controlan fuerza de trabajo, se mantienen relativamente constantes entre 1991 y 2001 , con un peso que oscila alrededor del $10 \%$.

TABLA 1

\begin{tabular}{|l|l|l|}
\hline $\begin{array}{l}\text { Distribución de la población ocupada en la rama agropecuaria según clase, } \\
\text { Argentina 1991-2001 }\end{array}$ \\
\hline Clase y fracción de clase & $\mathbf{1 9 9 1} \%$ & $\mathbf{2 0 0 1 \%}$ \\
\hline Gran burguesía & 0,1 & 0,1 \\
\hline Pequeños y medianos patrones & 9,3 & 8,7 \\
\hline Otros sectores de la pequeña burguesía acomodada & 1,8 & 1,7 \\
\hline Pequeños propietarios & 27,6 & 34,4 \\
\hline Otros sectores de la pequeña burguesía pobre & 0,5 & 0,6 \\
\hline Proletariado y semiproletariado & 60,7 & 54,48 \\
\hline Total & 100,0 & 100,00 \\
\hline
\end{tabular}

Elaboración a partir de datos producidos por el Programa de Investigación sobre el Movimiento de la Sociedad Argentina (PIMSA), sobre la base de datos de los Censos Nacionales de Población y Vivienda de 1991 y 2001 (Chazarreta y Rosati, 2016)

Un primer proceso que puede vincularse al análisis de estos datos, a partir de una mirada de largo plazo, es el modo en que el mismo desarrollo del capitalismo en el campo argentino alcanzó su límite en la incorporación de personas al trabajo en las actividades agrarias y ganaderas hacia la década del 50 y 60 . Luego, a partir de las décadas posteriores, el sector comenzó a expulsar población. Este proceso se profundizó a partir de los años 90, sobre todo con la intensificación tecnológica y los cambios organizativos y productivos que redujeron notablemente los requerimientos de fuerza de trabajo en buena parte de las actividades del sector. Si bien en la región pampeana los procesos de mecanización de las tareas de cosecha se habían producido hacia mediados del siglo XX, la adopción del paquete tecnológico vinculado a la soja y a las biotecnologías permitió reducir los requerimientos laborales en otras etapas del proceso productivo. A su vez, en otras regiones los procesos 
de mecanización de tareas sí se produjeron hacia la década del 90: el algodón constituye un caso relevante en este sentido.

Un segundo aspecto interesante es la disminución relativa del proletariado y semiproletariado, así como el crecimiento relativo de la pequeña burguesía pobre. No obstante, ambos grupos muestran un proceso de descenso en términos absolutos: el proletariado y semiproletariado disminuye aproximadamente un $40 \%$ y la pequeña burguesía pobre, alrededor del $16 \%$.

Este incremento en el peso de la pequeña burguesía pobre probablemente se vincule a procesos de diferenciación social de productores, en tanto podría estar expresando la constitución de ciertas capas de productores agrarios de pequeña escala en un contexto de concentración de la producción. Dos indicadores parecen aportar evidencia en este sentido: 1) la menor tasa de variación que presenta este grupo en relación con el resto y 2) que la región que mayor incremento relativo presenta del grupo de la pequeña burguesía pobre es el NEA (Chazarreta y Rosati, 2016, pp. 98-99). Así, por ejemplo, los casos de Chaco (Rosati, 2015) y Misiones (Schiavoni, 1998) pueden ser ilustrativos de este proceso.

El descenso en el peso del proletariado y semiproletariado puede estar vinculado tanto a la expulsión de población del sector agropecuario, ya mencionada, como a un incremento de la llamada "eventualidad" en el empleo agropecuario de parte de los asalariados. Estudios recientes han comprobado la alternancia entre el ingreso al mercado de fuerza de trabajo agropecuario, la desocupación y la inactividad (Rosati, 2019; Rau, 2012). Esto podría provocar problemas en la captación censal de estos contingentes de asalariados.

Estos datos hacen referencia al conjunto de la estructura social agraria argentina de manera agregada. Por ello, para estudiar y profundizar diferentes niveles de desarrollo del capitalismo es necesario dotar al análisis de un mayor nivel de desagregación. En ese sentido, cabe plantear las siguientes preguntas de investigación: ¿cuáles son las particularidades de la extensión y profundización del capitalismo en cada zona o área? ¿Cuáles son las diferentes estructuras agrarias que pueden identificarse en la formación social argentina? ¿Se pueden identificar patrones espaciales respecto a los tipos de estructuras agrarias o en realidad es aleatoria esa distribución? El presente trabajo abordará dichas preguntas. Constituye, en tal sentido, un primer ejercicio de estimación e identificación de dichas estructuras agrarias en función de la información estadística disponible.

Uno de los aportes fundamentales que se espera lograr es la construcción de una diferenciación de zonas o áreas que no se base en cercanía geográfica, como la categorización en regiones frecuentemente utilizada (Noroeste, Noreste, Patagonia, Pampeana y Cuyo), sino en la homogeneidad respecto a las estructuras sociales agrarias. Así, se parte de que "la región es simplemente un recurso conceptual, un construido, para tratar problemas específicos, teniendo que ser definida de nuevo ante cada problema" (Forni y Benencia, 1993, p. 4).

\section{Resultados PRincipales}

Como se ha señalado anteriormente, se trabajó con dos fuentes de datos diferentes, con unidades de análisis y definiciones operativas diferenciadas: mientras que el CNPyV releva información de la población (y en este caso específico, de la población ocupada), el CNA tiene como unidad de análisis las explotaciones agropecuarias.

No obstante, es evidente que en términos conceptuales ambos sets de variables remiten a una misma dimensión latente: las diferentes relaciones sociales productivas que pueden encontrarse en el sector agropecuario, o dicho de otra forma, las diferentes estructuras agrarias. Por ello, es esperable que existan correlaciones entre, por ejemplo, las variables vinculadas a la pequeña burguesía acomodada y los pequeños productores Tipo 1 (los más capitalizados) o tipo 2 (aquellos en la posición "intermedia").

A su vez, y dado que el objetivo de este trabajo es llegar a una clusterización de los diferentes departamentos que expresan diferentes estructuras agrarias, sería deseable poder reducir la dimensionalidad de este dataset. Por ello, se aplicará un Principal Components Analysis -PCA-. 
PCA es una técnica ampliamente utilizada para efectuar tareas de reducción de dimensionalidad. Básicamente, se parte de una matriz de $\mathrm{n} x \mathrm{p}$ (en nuestro caso, $492 \times 10$ ) y se busca obtener una nueva matriz $\mathrm{n} \times \mathrm{p}^{*}$ (donde $\mathrm{p}^{*}<\mathrm{p}$ ) de "componentes principales". Asumiendo (como es el caso) que las p variables se encuentran correlacionadas, los componentes principales ayudarán a resumir este conjunto de variables en un conjunto menor de variables que explicarán la mayor parte de la variabilidad del dataset original. ${ }^{9}$ Para ello (y dado que se trata de datos composicionales), se transformaron las proporciones tomando sus raíces cuadradas, se las centró en la media y se escaló utilizando el desvío estándar.

TABLA 2

\begin{tabular}{|l|l|l|l|l|l|}
\hline Varianza explicada por los componentes principales \\
\hline & PC1\% & PC2\% & PC3 \% & PC4\% & PC5\% \\
\hline \% Varianza explicada & 40,00 & 17,73 & 13,36 & 9,94 & 5,89 \\
\hline \% Varianza explicada & 40,00 & 57,77 & 71,13 & 81,07 & 86,95 \\
\hline acumulada
\end{tabular}

Elaboración propia sobre la base de CNPyV 2001 y datos cedidos por Obschatko, Foti y Román (2007)

Con 5 componentes es posible retener el $87 \%$ de la varianza contenida en el set de 10 variables originales.

FIGURA 1

Correlaciones entre los 5 componentes principales y las variables originales

\begin{tabular}{|c|c|c|c|c|c|}
\hline patt - & -0.56 & 0.6 & -0.04 & 0.04 & -0.15 \\
\hline D_pys - & -0.64 & -0.55 & -0.13 & -0.49 & 0.1 \\
\hline P_PPT3EAP - & 0.77 & -0.38 & 0.41 & -0.03 & -0.06 \\
\hline P_PPT2EAP - & 0.25 & 0.55 & 0.18 & -0.61 & -0.06 \\
\hline$\Sigma_{\bar{N}}^{\text {P_PPT1EAP - }}$ & -0.32 & 0.73 & -0.22 & -0.02 & 0.14 \\
\hline D_POP_prop - & 0.82 & 0.34 & 0.07 & 0.42 & -0.03 \\
\hline P_Dop_aux - & -0.54 & -0.11 & 0.48 & 0.1 & -0.32 \\
\hline D_pba_ofic- & -0.35 & 0.02 & 0.59 & 0.21 & 0.58 \\
\hline P_pba_aux- & -0.56 & 0.07 & 0.54 & 0.22 & -0.22 \\
\hline P_NOPPEAP - & 49.78 & -0.18 & -0.41 & 0.31 & 0.02 \\
\hline & P'1 & PC2 & $\begin{array}{l}\mathrm{PC} 3 \\
\text { var2 }\end{array}$ & $\mathrm{PC}_{4}$ & P'́5 \\
\hline
\end{tabular}

Elaboración propia sobre la base de CNPyV 2001 y datos cedidos por Obschatko, Foti y Román (2007) Notas: patr: prop. patrones, p_pys: prop. proletariado y semiproletariado,

P_PPT3EAP: prop. EAPS de peq. productores

T3, P_PPT2EAP: prop. EAPS de peq. productores

T2, P_PPT1EAP: prop. EAPS de peq. productores

p_ppb_prop: prop. pequeños patrones (peq. burguesía pobre), p_ppb_aux: prop. peq. burguesía pobre asalariada,

p_pba_ofic y p_pba_aux: prop. pequeña burguesía acomodada asalariada,

P_NOPPEAP: prop. EAPS de no pequeños productores

A su vez, al analizar los pesos de los componentes y la matriz de correlaciones puede notarse

- que el componente 1 parece captar la presencia de PP tipo 3 (es decir, los que menores capacidades de reproducción ampliada poseen) y también aparece con una correlación alta en el tipo social análogo en el CNPyV, es decir, los pequeños propietarios (capa de la pequeña burguesía pobre); 
- que el componente 2 presenta altas correlaciones en las variables asociadas a la gran burguesía y a las capas acomodadas de la pequeña burguesía (particularmente, los pequeños patrones); además, consistentemente, presenta altas correlaciones en el tipo 1 (el más capitalizado) de pequeños productores y, en menor medida, con el tipo 2;

- que el componente 3 presenta correlaciones mayores en las capas asalariadas de la pequeña burguesía tanto acomodada como pobre;

- que el componente 4 parece asociado de forma negativa a los estratos medios (tipo 2) de pequeños productores y positivamente (aunque en menor medida) a las capas de pequeños propietarios de la pequeña burguesía, y

- que el componente 5, por último, aparece más asociado a la capa de asalariados de la pequeña burguesía acomodada

\subsection{Construyendo las estructuras agrarias: clustering}

Para construir las estructuras se utilizó el llamado clustering o análisis de conglomerados. Es una técnica estadística multivariante que busca agrupar elementos, en este caso departamentos, tratando de lograr la máxima homogeneidad en cada grupo y la mayor diferencia entre los grupos (James, Witten, Hastie y Tibshirani, 2017). Su objetivo principal es clasificar objetos en grupos lo más homogéneos posible en relación con el conjunto de variables consideradas, de manera tal que cada registro pertenezca a un grupo y solamente a uno, y el conjunto de dichos grupos contenga a todos los objetos. La métrica utilizada puede ser una distancia por la cual los grupos formados contendrán individuos parecidos de forma que la distancia entre ellos ha de ser pequeña (por ejemplo, la distancia euclídea) o una similaridad por la cual los grupos formados contendrán individuos con una similaridad alta entre ellos (por ejemplo, el coeficiente de correlación).

En este trabajo se utilizó el método de clustering jerárquico. La matriz de distancias fue calculada utilizando distancias de tipo Manhattan y el método de aglomeración utilizado fue el de Ward. ¿Cuál es el número óptimo de clusters para construir? Para eso se utilizó el gap statistic ${ }^{10}$ (Hastie, Walther y Hastie, 2001):

FIGURA 2

Gap statistic para la determinación del número de clusters

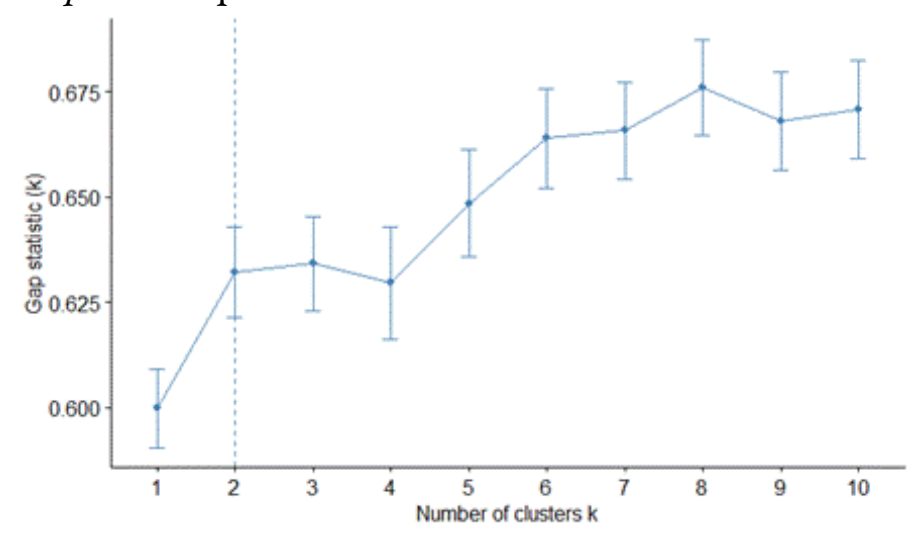

Elaboración propia sobre la base de CNPyV 2001 y datos cedidos por Obschatko, Foti y Román (2007)

El número óptimo parece ser dos clusters. No obstante, puede verse que no existen pérdidas significativas de performance al incrementar levemente la cantidad. De hecho, al analizar el dendograma, parecen conformarse cuatro clusters: 


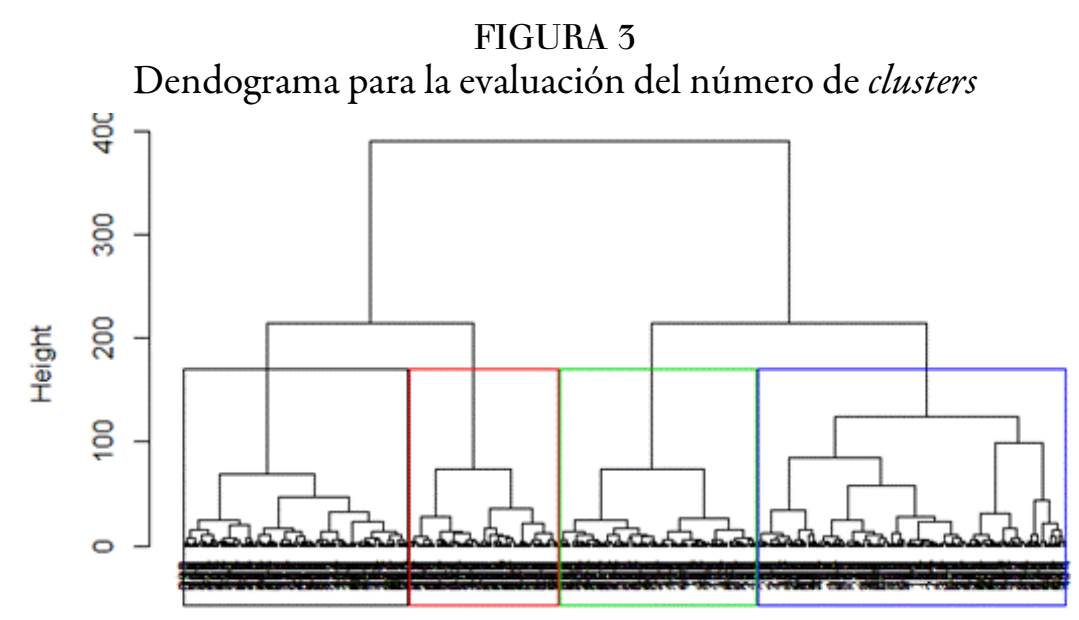

Elaboración propia sobre la base de CNPyV 2001 y datos cedidos por Obschatko, Foti y Román (2007)

\subsection{Interpretación de los clusters}

¿Qué características tienen los clusters conformados? ¿Qué tipo de estructuras agrarias concretas identifican?Para caracterizar e interpretar cada uno de los clusters (que se corresponde con un tipo de estructura agraria), se calculó la mediana de cada una de las variables originales al interior de cada uno de los conglomerados:

\section{FIGURA 4}

Métricas para la caracterización de cada cluster. Mediana de cada variable para cada cluster

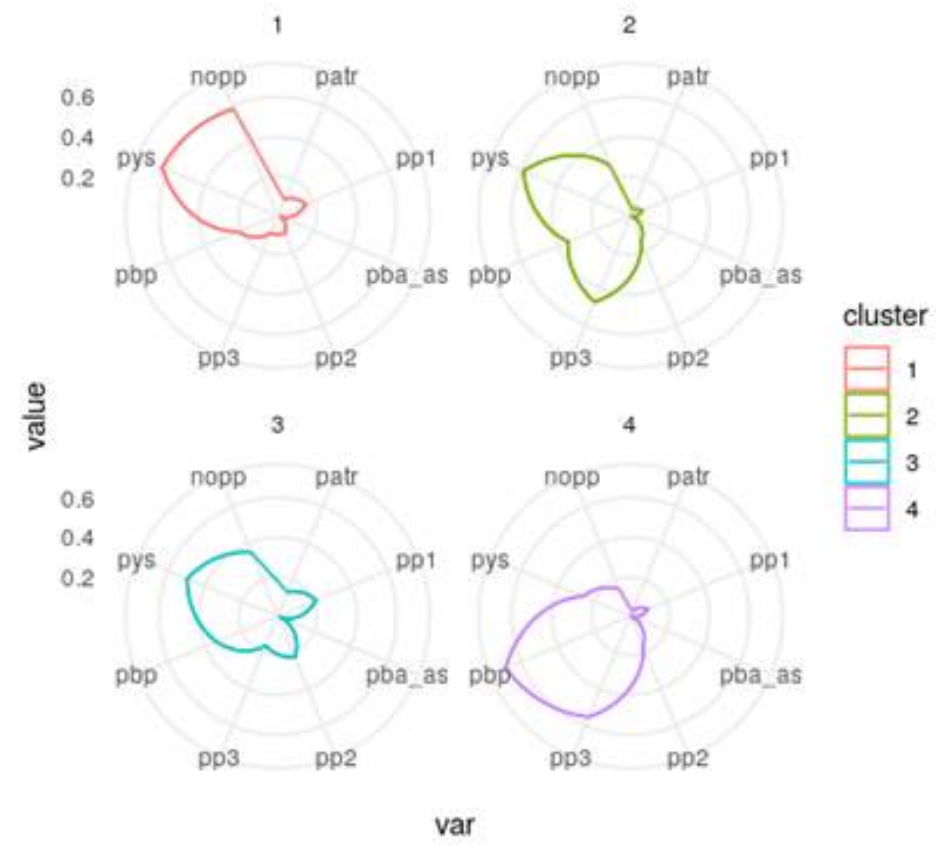

Elaboración propia sobre la base de CNPyV 2001 y datos cedidos por Obschatko, Foti y Román (2007) patr: prop. Patrones, pp1: prop. peq. productores T1,

pba_as: prop. peq. burguesía acomodada asalariada, pp2: prop. peq. productores T2, pp3: prop. peq. productores T3, ppb: prop. peq. burguesía pobre, pys: prop. proletariado y semiproletariado, nopp: prop de productores "no pequeños". 
El cluster 1 se caracteriza por una elevada proporción de población proletaria y semiproletaria en el campo, así como por una elevada proporción de EAP's que son consideradas como de "no pequeños productores"y de pequeños productores "capitalizados". Los tres son indicadores de un alto desarrollo de las relaciones salariales en dicha estructura: por un lado, alta proporción de trabajo asalariado, alta prevalencia de explotaciones grandes y de explotaciones "pequeñas" pero con capacidad de acumulación, es decir, cercanas a fracciones de pequeña burguesía acomodada. Por tanto, este cluster lo conceptualizamos como alto grado de desarrollo de relaciones salariales. Ejemplos de este tipo de estructuras son los departamentos del centro y sureste de la provincia de Buenos Aires que concentran buena parte del stock ganadero nacional, como los partidos de General Lavalle y Olavarría.

El cluster 2 también presenta un alto grado de desarrollo de las relaciones salariales (expresado en la proporción alta de proletariado y semiproletariado). Pero su característica específica es que se combinan con una igualmente elevada prevalencia de pequeños productores de tipo 3. Estos productores, según la clasificación de IICA, son aquellos que cuentan con una escasa cantidad de medios de vida -tierra y equipamiento- y se encuentran potencialmente incapacitados de realizar la reproducción simple de su explotación. De esta forma, esta estructura muestra la presencia y articulación de amplias capas de trabajadores agropecuarios expropiados con pequeños productores cercanos a capas de semiproletariado, que no pueden vivir exclusivamente de su producción. De esta forma, identificamos esta estructura como de un alto grado de desarrollo de las relaciones salariales y existencia de fracciones de superpoblación latente en el campo. Ejemplos de este tipo de estructuras son algunos departamentos localizados en las provincias norteñas de Jujuy (como El Carmen y Ledesma), Salta (como Orán y General Güemes) y Tucumán (como La Cocha y Alberdi), dedicados especialmente a cultivos industriales como caña de azúcar y tabaco.

A su vez, el cluster 3 muestra una mayor proporción de proletariado y semiproletariado (es decir, un alto grado de desarrollo de relaciones salariales). La diferencia con el tipo 2 es que la articulación que se observa es con explotaciones de pequeños productores tipo 1 y tipo 2. Estos tipos de productores estaban caracterizados, según el IICA, por la posibilidad de lograr acumulación en escala ampliada (tipo 1) y en escala simple (tipo 2). Se trata, entonces, de una estructura agraria caracterizada por un alto desarrollo de relaciones salariales articuladas con fracciones de pequeños propietarios simples y acomodados. Ejemplos de este tipo de estructuras son algunos departamentos localizados en el sur y este de la provincia de Santa Fe (como Iriondo y Belgrano) y casi la totalidad de los ubicados en la provincia de Entre Ríos (Nogoyá y Gualeguaychú), caracterizados por la importancia de la producción de soja.

Por último, el cluster 4 presenta como rasgos salientes una mayor prevalencia de pequeños productores pobres tipo 3 y de fracciones de pequeña burguesía pobre. Se trataría de una estructura basada en la pequeña propiedad, con bajo grado de desarrollo de relaciones salariales y con una prevalencia importante de pequeños productores pobres, es decir, una estructura basada en la pequeña producción mercantilsimple con elementos de descomposición. Ejemplos de este tipo de estructuras son casi todos los departamentos localizados en Formosa y los departamentos ubicados al norte y al noroeste de Chaco, entre ellos, los que se sitúan en la región de El Impenetrable. 
TABLA 3

\begin{tabular}{|c|c|c|c|c|}
\hline \multicolumn{5}{|c|}{$\begin{array}{l}\text { Indicadores de pluriactividad bajo categoría ocupacional asalariada para cada } \\
\text { estructura }\end{array}$} \\
\hline \multirow[b]{2}{*}{ Estructura } & \multicolumn{2}{|c|}{$\begin{array}{l}\text { Sobre total de } \\
\text { pluriactivos en cada } \\
\text { categoría }\end{array}$} & \multicolumn{2}{|c|}{$\begin{array}{l}\text { Sobre el total de EAPS de } \\
\text { peq. prod en cada } \\
\text { estructura }\end{array}$} \\
\hline & \begin{tabular}{|l|} 
En el sector \\
agropecuario \\
$\%$
\end{tabular} & \begin{tabular}{|l|} 
Fuera del \\
sector \\
agropecuario \\
$\%$
\end{tabular} & \begin{tabular}{|l|} 
En el sector \\
agropecuario \\
$\%$
\end{tabular} & \begin{tabular}{|l|} 
Fuera del \\
sector \\
agropecuario \\
$\%$
\end{tabular} \\
\hline $\begin{array}{l}\text { 1- Alto grado de desarrollo } \\
\text { capitalista }\end{array}$ & 16.7 & 12.4 & 6.29 & 7.70 \\
\hline $\begin{array}{l}\text { 2- Alto grado de desarrollo } \\
\text { cisuperpoblación relativa }\end{array}$ & 34.4 & 39.5 & 6.88 & 13.05 \\
\hline $\begin{array}{l}\text { 3- Alto desarrollo c/pequeña } \\
\text { producción capitalista y } \\
\text { mercantil simple }\end{array}$ & 27.0 & 18.3 & 4.93 & 5.49 \\
\hline $\begin{array}{l}\text { 4- Pequeña producción } \\
\text { mercantil simple en } \\
\text { descomposición }\end{array}$ & 21.9 & 29.8 & 4.03 & 9.04 \\
\hline
\end{tabular}

Elaboración propia sobre la base de datos cedidos por Obschatko, Foti y Román (2007)

Un indicador útil para realizar una primera validación de estas estructuras es la proporción de productores que realizan trabajos extraprediales. Si bien las formas de la llamada "pluriactividad" son múltiples, en general la presencia de trabajos extraprediales bajo la forma asalariada dentro, pero sobre todo, fuera del sector agropecuario ha sido conceptualizada clásicamente (Lenin, 1975; Kautsky, 1974) como indicadores de la existencia de procesos de descomposición campesina, de diferenciación social e incluso de constitución de fracciones de superpoblación en el campo. Al observar la distribución de la pluriactividad bajo la forma asalariada de productores que pertenecen a alguno de los tres tipos de productores agropecuarios, podemos ver que las estructuras 2 y 4 (caracterizadas por la presencia de superpoblación relativa y pequeña producción en descomposición) concentran la mayor parte de las explotaciones de pequeños productores que trabajan como asalariados fuera del sector agropecuario (casi el $70 \%$ del total) y dentro del sector (56\%). A su vez, la estructura 3 tiene un peso relevante en este último indicador (27\%). Las estructuras 2 y 4 muestran, al mismo tiempo, una mayor prevalencia de pequeños productores pluriactivos bajo la forma asalariada que el resto, espacialmente fuera del sector agropecuario: la estructura 2 presenta un $13 \%$ de pequeños productores en esta situación y la estructura 4 , un $9 \%$. 
FIGURA 5

Indicadores seleccionados para cada estructura agraria. Argentina, 2001-2002

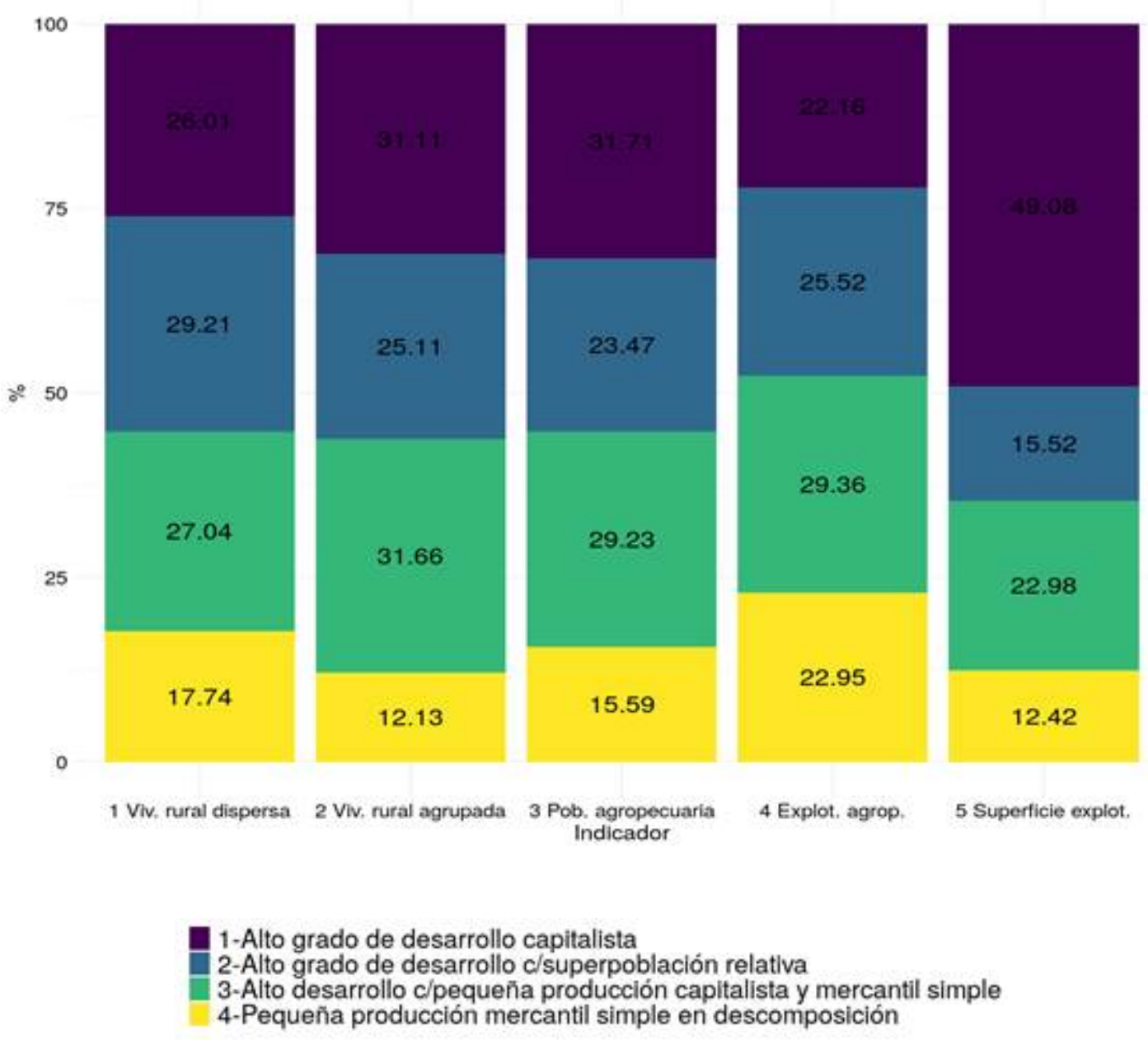

Elaboración propia sobre la base de CNPyV 2001 y datos cedidos por Obschatko, Foti y Román (2007)

En el gráfico anterior pueden verse algunos indicadores seleccionados que permiten dar una noción de la escala que tiene cada una de las estructuras agrarias identificadas. Las estructuras 1 y 3 concentran la mayor parte de las viviendas en zonas rurales agrupadas (ambas el $31 \%$ ), mientras que las viviendas en zonas rurales dispersas aparecen más distribuidas en las estructuras 1,2 y 3 (26\%, $29 \%$ y $27 \%$, respectivamente). Al observar la población agropecuaria, puede verse que casi un tercio se encuentra en la estructura de capitalismo desarrollado (1) y otro tanto en estructura 2 (29\%). Las explotaciones, en cambio, aparecen distribuidas de forma más homogénea a lo largo de las diferentes estructuras. A su vez, la mitad de la superficie de las explotaciones agropecuarias aparece ubicada en la estructura 1.

Al observarse la distribución geográfica (figura 6), en primer lugar puede notarse la extensión geográfica que abarca el desarrollo de relaciones capitalistas en el campo. La gran mayoría de los departamentos presentan estructuras con alto grado de relaciones salariales. No obstante, parte de ellas se articulan con otros dos componentes: 1) pequeña producción mercantil y pequeña producción capitalista (norte y centro-oeste de la provincia de Buenos Aires, sur, centro y oeste de Santa Fe, zona algodonera chaqueña, etc.) y 2) fracciones de superpoblación relativa (observables en la existencia de explotaciones de pequeños productores tipo 3 ).

A su vez, se observa que existe un área significativa (de alrededor del $12 \%$ ) de la formación social del campo argentino que concentra estructuras vinculadas a la pequeña producción mercantil simple (que otros estudios 
podrían caracterizar como campesinas). En algunos de estos departamentos se observa la combinación con pequeños productores de tipo 1 o 2 .

\section{FIGURA 6}

Distribución de las estructuras agrarias identificadas a nivel departamento, Argentina 2001-2002

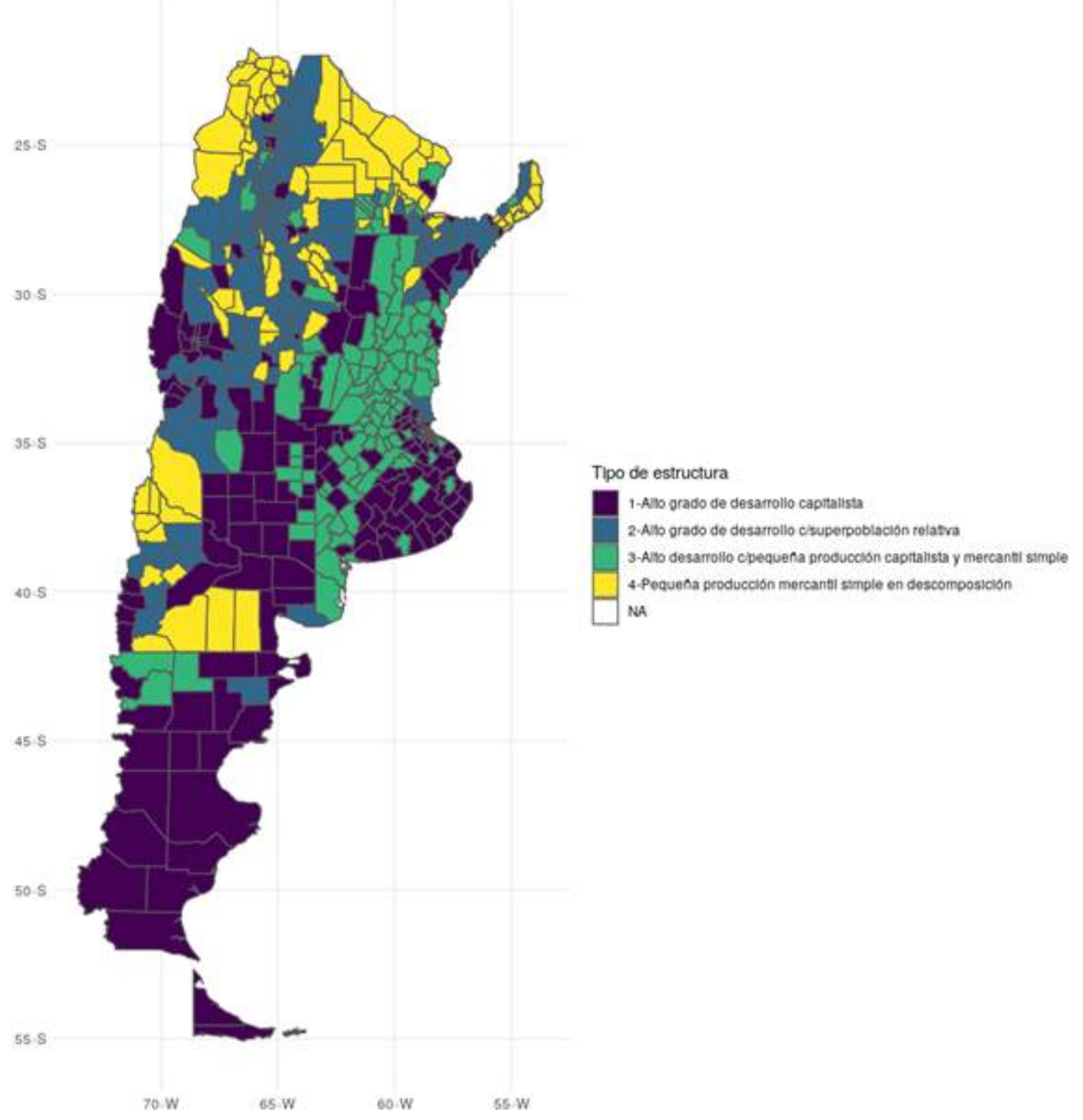

Elaboración propia sobre la base de CNPyV 2001 y datos cedidos por Obschatko, Foti y Román (2007) El mapa en formato interactivo se encuentra disponible para un análisis exploratorio en el siguiente link: https://gefero.github.io/mapa_estr/.

Sin embargo, en otros departamentos se observa la existencia de articulación con pequeños productores de tipo 3. Es decir, parte de dicha estructura parece encontrarse en crisis, en tanto las explotaciones que operan en ella no parecen lograr niveles de reproducción simple.

Cabe mencionar, finalmente, que si bien existe un cierto grado de autocorrelación espacial, no deja de ser cierto que se observan saltos y discontinuidades en la distribución de las estructuras agrarias. No se trata de una distribución aleatoria, pero tampoco de un gradiente uniforme. Tales discontinuidades quedarían oscurecidas si solamente se tratara de regionalizar en función de criterios ambientales o geográficos. 


\section{Discusión Y NUEvos PROBLEMAS}

En este trabajo se intentó realizar un primer ejercicio de identificación de las estructuras agrarias existentes en la Argentina entre 2001 y 2002. Para ello se utilizaron datos censales de población y agropecuarios a nivel departamental y se utilizaron técnicas de análisis multivariado (PCA y clustering jerárquico) para su detección.

Se observa la existencia de cuatro estructuras agrarias, tres de las cuales presentan rasgos de alto desarrollo de relaciones salariales conjuntamente con elementos de pequeña producción mercantil simple, pequeña producción de carácter capitalista y elementos de superpoblación relativa. La estructura restante muestra una articulación entre pequeña producción mercantil simple con rasgos de descomposición.

Ahora bien, este primer ejercicio muestra que es viable la incorporación de dimensiones vinculadas a la estructura social agraria para lograr regionalizaciones que permitan ampliar los indicadores geográficos y productivos, que son los utilizados habitualmente. El carácter exploratorio del presente ejercicio hace necesario marcar algunas líneas sobre las que sería posible ampliar sus alcances:

- realizar una optimización más fina de los hiperparámetros del modelo seleccionado: incluir otras métricas de distancia, otros métodos de aglomeración, etc.; ${ }^{11}$

- incorporar mayor cantidad de indicadores provenientes de los CNA (cantidad de asalariados permanentes y jornadas contratadas por transitorios) e incluso información de los Núcleos de Agricultura Familiar provenientes del Registro Nacional de la Agricultura Familiar (ReNAF);

- analizar los diferentes patrones de uso del suelo en cada una de las estructuras agrarias identificadas; particularmente, en relación con la predominancia de cultivos con diferentes niveles de desarrollo y paquetes tecnológicos; $\mathrm{y}$

- vinculado con el punto anterior, incluir un análisis de las distancias de cada departamento a los principales puertos y puntos de comercialización.

Asimismo, se espera ampliar el alcance temporal del presente ejercicio. En efecto, sería posible replicar y adaptar la metodología de identificación de las estructuras agrarias en el período 1988-1991 utilizando información del CNA 1988 y del CNPyV 1991. Como se ha planteado más arriba, también se evaluará la posibilidad de identificar las estructuras agrarias en un período más reciente.

También resulta relevante mencionar que la construcción de este tipo de agregaciones de unidades geográficas (departamentos e incluso unidades menores, si fuera permitido por la forma de presentación de la información) permite el replanteo de algunos temas instalados en la literatura de los estudios rurales. Los resultados obtenidos (y los que surjan de la aplicación de esta metodología en censos más recientes) deberían permitir un análisis más detallado de algunos aspectos vinculados a otras dimensiones desarrolladas del capitalismo en el campo. ¿En qué estructuras agrarias se produce la expansión de la frontera agrícola llevada adelante por cultivos como la soja? ¿Es posible periodizar dicho movimiento? Similares preguntas podrían plantearse respecto de los procesos de deforestación.

Al mismo tiempo, existe en la literatura la imagen del proceso de sojización como responsable de la desaparición de explotaciones. En este sentido, algunos resultados preliminares sugieren que el proceso de desaparición de explotaciones agropecuarias varía según el tipo de estructura social agraria en el que se desarrolla la expansión de la frontera y que tal tipo de estructura agraria tiene una influencia comparable a los procesos de expansión.

Finalmente, la combinación con otras fuentes de datos debería permitir especificar problemas e hipótesis que vinculen la dinámica agropecuaria con las que se producen en otros territorios productivos. ¿Hasta qué punto el desarrollo de actividades de enclave (como minería, extracción de petróleo, etc.) coincide con ciertas particularidades de las estructuras sociales agrarias identificadas? 


\section{REFERENCIAS}

Archetti, Eduardo y Stölen, Kristi (1975). Explotación familiar y acumulación de capital en el campo argentino. Buenos Aires: Siglo XXI.

Balsa, Javier (2006). El desvanecimiento del mundo chacarero. Transformaciones sociales en la agricultura bonaerense, 1937-1988. Bernal: Universidad Nacional de Quilmes.

Bartolomé, Leopoldo (1975). Colonos, plantadores y agroindustrias. La explotación agrícola familiar en el sudeste de Misiones. Desarrollo Económico, 15 (58), 239-264.

Barsky, Andrés (S/F). Propuesta metodológica de zonificación agraria de la Pampa argentina a partir de los datos del Censo Nacional Agropecuario 1988. Recuperado de http://www.observatoriogeograficoamericalatina.org.mx/ egal6/Geografiasocioeconomica/Geografiaagraria/408.pdf.

Barsky, Andrés (1997). La puesta en valor y producción del territorio como generadora de nuevas geografías. Propuesta metodológica de zonificación agroproductiva de la Pampa Argentina a partir de los datos del Censo Nacional Agropecuario 1988. En O. Barsky y A. Pucciarelli (Editores), El agro pampeano. El fin de un periodo (pp. 405-482). Buenos Aires: FLACSO/CBC.

Barsky, Osvaldo, Pucciarelli, Alfredo y Barsky, Andrés (1997). Configuración espacial de los principales ámbitos regionales agrarios del país. En O. Barsky y A. Pucciarelli (Editores): El agro pampeano. El fin de un periodo (pp. 361-403). Buenos Aires: FLACSO/CBC.

Bertamini, Felipe, Bervejillo, José, Silva, María y Tommasino, Humberto (S/F). Regionalización agropecuaria según estructura del valor de la producción. Estudios de Economía Agraria y Ambiental, 15(03). Recuperado de https://www.gub.uy/ministerio-ganaderia-agricultura-pesca/sites/ministerio-ganaderia-agricultura-pesca/fi les/documentos/publicaciones/e_15_03_vbp_regional_jb_mes_ht_fb.pdf

Bisio, Raúl y Forni, Floreal (1980). Empleo rural en la República Argentina 1937-1969. Documento de Trabajo № 1. Buenos Aires: CEIL.

Chazarreta, Adriana, Poth, Carla y Ramírez, Delia (2015). Dinámicas estatales en la inserción de la globalización de la agricultura en Argentina: tensiones y recomposiciones institucionales. En M. Svampa (Comp.), El desarrollo en disputa. Actores, conflictos y modelos en la Argentina contemporánea (pp. 111-151). Buenos Aires: Editorial UNGS.

Chazarreta, Adriana y Rosati, Germán (2016). Los cambios en la estructura social agraria argentina (1988-2012). En G. Kessler (Comp.), La sociedad argentina hoy. Radiografia de una nueva estructura (pp. 85-107). Buenos Aires: Siglo XXI.

Cirio, Félix (1984). Evolución reciente y perspectivas de crecimiento de la agricultura de la región pampeana. Documento $\mathrm{n}^{\circ}$ 8. Buenos Aires: CISEA.

CONADE-CFI (1968). Tenencia de la tierra. Aspectos de la estructura agraria y su incidencia en el desarrollo agropecuario argentino. Buenos Aires: CFI.

Donaire, Ricardo y Rosati, Germán (2009). "Evolución de la distribución de la población según grupos sociales fundamentales", Documento de Trabajo n68, en PIMSA. Documentos y Comunicaciones 2008/09 (pp. 8-22). Buenos Aires: : PIMSA.

Donaire, Ricardo y Rosati, Germán (2010). "Estructuras económico sociales concretas que constituyen la formación económica de la Argentina 1980-2001”, Documento de Trabajo n 76, en PIMSA. Documentosy Comunicaciones 2010 (pp. 50-103). Buenos Aires: PIMSA.

Forni, Floreal y Benencia, Roberto (1993). Las relaciones entre empleo, producción y población en el agro argentino entre 1914 y 1969. Documento de Trabajo N³4. Buenos Aires: CEIL.

Gómez, Pedro, Peretti, Miguel, Pizarro, José y Cascardo, Antonio (1991). Delimitación y caracterización de la región. En O. Barsky (Ed.), El desarrollo agropecuario pampeano (pp. 77-94). Buenos Aires: Grupo Editor Latinoamericano. 
Gras, Carla y Cáceres, Daniel (2017). El acaparamiento de tierras como proceso dinámico. Las estrategias de los actores en contextos de estancamiento económico. Población y Sociedad, 24(2). Recuperado de https://cerac.unlpam.e du.ar/index.php/pys/article/view/2831/2907.

James, Gareth, Witten, Daniela, Hastie, Trevor y Tibshirani, Robert (2017). An Introduction to Statistical Learning with Applications in R. Berlín: Springer.

Iñigo Carrera, Nicolás (2011). Génesis, formación y crisis del capitalismo en el Chaco, 1870-1970. Salta: Universidad Nacional de Salta

Iñigo Carrera, Nicolás y Podestá, Jorge (1985). “Análisis de una relación de fuerzas sociales objetiva: caracterización de los grupos sociales fundamentales en la Argentina actual”. Documento de Trabajo Serie Estudios n 46. Buenos Aires: CICSO.

Iñigo Carrera, Nicolás, Podestá, Jorge y Cotarelo, María Celia (1999). "Las estructuras económico-sociales que constituyen la formación social argentina”, Documento de Trabajo n 18 en PIMSA. Documentos y Comunicaciones 1999 (pp. 37-81). Buenos Aires: PIMSA.

Kautsky, Karl (1974). La cuestión agraria. Buenos Aires: Siglo XXI.

Lenin, Vladimir (1975). El desarrollo del capitalismo en Rusia. El proceso de la formación del mercado interior para la gran industria. Moscú: Ed. Progreso.

Lenin, Vladimir (1983). El programa agrario de la socialdemocracia rusa en la primera revolución rusa de 1905-1907. En Obras completas. Tomo 16 (pp. 201-440). Moscú: Ed. Progreso.

Lenin, Vladimir (1985). Nuevos datos sobre las leyes de desarrollo del capitalismo en la agricultura. En Obras completas. Tomo 27 (pp. 135-238). Moscú: Ed. Progreso.

Marx, Karl (2005). El Capital. Tomo I. Buenos Aires: Siglo XXI.

Margiotta, Edgardo y Benencia, Roberto (1988). Introducción al estudio de la estructura agraria. Buenos Aires: Facultad de Agronomía, Universidad Nacional de Buenos Aires, mimeo.

Obschatko, Edith (2009). Las explotaciones agropecuarias familiares en la República Argentina: un análisis a partir de los datos del Censo Nacional Agropecuario 2002. Documento de Trabajo Serie Estudios e Investigaciones n²3. Buenos Aires: Ministerio de Agricultura, Ganadería y Pesca-IICA.

Obschatko, Edith, Foti, María del Pilar y Román, Marcela (2007). "Los pequeños productores en la República Argentina: importancia en la producción agropecuaria y en el empleo en base al censo nacional agropecuario 2002", Documento de Trabajo Serie Estudios e Investigaciones n¹0, Buenos Aires: SAGPyA-IICA.

Obschatko, Edith, Soverna, Susana y Tsakoumagkos, Pedro (2016). Las explotaciones agropecuarias empresariales en la Argentina. Buenos Aires: IICA.

Rau, Víctor (2012). Cosechando yerba mate. Estructuras sociales de un mercado laboral agrario en el Nordeste argentino. Buenos Aires: CICCUS.

Rosati, Germán (2013). Patrones espaciales de expansión de la frontera agrícola: la soja en la Argentina (1987-1988/2009-2010). En C. Gras y V. Hernández (Comps.), El agro como negocio: producción, sociedad y territorios en la globalización (pp. 97-122). Buenos Aires: Biblos.

Rosati, Germán (2015). Funcionalidad y diferenciación en un mercado de fuerza de trabajo en metamorfosis. El caso de la cosecha de algodón en Chaco (1960-2010) (Tesis doctoral, inédita). Universidad Nacional de Buenos Aires, Buenos Aires.

Rosati, Germán (2019). Ciclos de ocupación anuales y patrones de movilidad- de los trabajadores agropecuarios: una aproximación a sus determinantes mediante técnicas de Ensamble Learning. Estudios Rurales, 10(9). Recuperado de http://ppct.caicyt.gov.ar/index.php/estudios-rurales/article/view/16878/45454575770133.

Schiavoni, Gabriela (1998). Colonos y ocupantes. Parentesco, reciprocidad y diferenciación social en la frontera agraria de Misiones. Posadas: Editorial Universitaria de Misiones.

Secretaría de Agricultura, Ganadería y Pesca (1987). "Regionalización agroeconómica de la Argentina”, Estudio para la implementación de la Reforma Impositiva Agropecuaria, Proyecto PNUD Argentina 85/019. 
Slutzky, Daniel (1968). Aspectos sociales del desarrollo del desarrollo rural en la Pampa Húmeda, Desarrollo Económico, 9(9), 95-135.

Taylor, Carl (1948). Rural life in Argentina. Louisana: Louisana State University Press.

Tibshirani, Robert, Walther, Guenter y Hastie, Trevor (2001). Estimating the number of data clusters in a data set via the Gap statistic. Journal of the Royal Statistical Society, 63(2), 411-423.

Vicien, Jorge y Dejean del Castillo, Raúl (1948). Regiones social-agrarias de la República Argentina. Buenos Aires: Ministerio de Agricultura de la Nación.

\section{Notas}

1 Una versión preliminar de este trabajo fue presentada en las XI Jornadas Interdisciplinarias de Estudios Agrarios y Agroindustriales Argentinos y Latinoamericanos, realizadas entre el 5 y el 8 de noviembre de 2019 en la Facultad de Ciencias Económicas de la UBA

2 Eventualmente, se evaluará la posibilidad de extender el ejercicio para el período posterior, que se corresponde con el Censo Nacional de Población, Hogares y Vivienda del año 2010 y el Censo Nacional Agropecuario de 2018, cuyos datos preliminares recién se estaban publicando al momento de escribir el presente artículo. El Censo de Población de 2010 no codificó de forma completa la variable ocupación: está codificada sólo a dos dígitos; por lo tanto, no es posible conocer la calificación (cuarto dígito), característica necesaria para construir los grupos sociales. A su vez, existe un relevamiento censal agropecuario previo al 2018 (el Censo Nacional Agropecuario de 2008), pero el operativo de campo se desarrolló en una coyuntura signada por el conflicto por las retenciones móviles, por lo cual parece haber presentado una serie de problemas que es necesario indagar antes de utilizar dicha fuente.

3 Existe una gran diversidad de temas y análisis en los estudios rurales y agrarios argentinos que se vinculan de alguna manera a las formas de desarrollo del capitalismo en el campo. De esta forma, los procesos de génesis histórica de las diferentes fracciones de la fuerza de trabajo del sector y sus transformaciones recientes ilustran dimensiones vinculadas a la formación de las estructuras agrarias concretas en la Argentina. No obstante, dada su especificidad no son reseñados en este trabajo.

4 Ejercicios similares pero con un nivel de desagregación provincial para el total de la formación social argentina (y no sólo para su sector agropecuario) pueden encontrarse en Iñigo Carrera, Podestá y Cotarelo (1999) y Donaire y Rosati (2010).

5 En este estudio también se incorporan para la regionalización otras variables como valor de la producción agropecuaria y distribución de la tierra.

6 Para mayor detalle acerca de los criterios técnico-metodológicos para la construcción de grupos sociales fundamentales, ver Donaire y Rosati (2009).

7 Una característica de la pequeña burguesía acomodada es el hecho de que su escala de acumulación implica que los mismos propietarios y productores deban participar de forma directa en el proceso productivo y no cumpliendo exclusivamente la función de capital. Esto no modifica el hecho fundamental de que constituyen fracciones de población que son propietarias de sus propios medios de vida y de medios de vida para otros, aun cuando se trate de "pequeños patrones" (Marx, 2005, p. 374). Quizás sea esta característica de la pequeña burguesía la que explica en parte la proliferación de categorías descriptivas en los estudios agrarios que buscan hacer patente este hecho (farmers, colonos, etc.).

8 En este estudio se define a los pequeños productores como aquellos productores agropecuarios que dirigen una explotación agropecuaria (EAP) en la que el productor agropecuario trabaja directamente en la explotación; no se contratan trabajadores no familiares remunerados permanentes y no tienen como forma jurídica la sociedad anónima o en comandita por acciones. Por último, los límites de superficie total o cultivada varían en función de la provincia (Obschatko, Foti y Román, 2007, pp. 32 y 33).

9 Para mayor información al respecto, puede revisarse James, Witten, Hastie y Tibshirani (2017).

10 El gap statistic compara la variabilidad total intra-cluster para diferentes valores de $\mathrm{k}$ (número de clusters) contra sus valores esperados bajo una distribución esperada de referencia. El k óptimo será el que maximice el gap statistic, lo cual supone que la estructura de clusterización que se encuentra está lejos de una distribución uniforme de puntos.

11 No obstante, es necesario resaltar que en una exploración preliminar (con otras métricas de distancia, como por ejemplo, distancia euclidiana), los resultados obtenidos parecen ser bastante robustos. 